\section{Constitution of Conessine}

Previous work has shown that the alkaloid, conessine, is a ditertiary base, $\mathrm{C}_{24} \mathrm{H}_{40} \mathrm{~N}_{5}$, containing three $\mathrm{N}$-methyl groups and one ethylenic linkage, and our work now establishes a relationship with the growing class of steroid bases. The absence of oxygen is noteworthy; this is uncommon in the alkaloids, and the natural occurrence of an oxygen-free steroid derivative has not been reported previously.

The first indication of the structure came from an examination of the selenium dehydrogenation products of the hydrocarbon mixture, $\mathbf{C}_{21} \mathbf{H}_{30}$, obtained by pyrolysis of conessine dihydriodide ${ }^{1}$. Careful fractionation of the products yielded a crystalline hydrocarbon, $\mathrm{C}_{18} \mathrm{H}_{18}$, m.p. 78-79 ${ }^{\circ}$ which showed many similarities with synthetic ${ }^{2} 3^{\prime}$-ethylcyclopentenophenanthrene $(\mathrm{I})$, m.p. $84 \cdot 5-85 \cdot 5^{\circ}$.

Although the hydrocarbons and their derivatives with nitrohydrocarbons gave no depression in melting point on admixture, it was not possible to raise the melting point of the hydrocarbon obtained from conessine, even after recovery from the nitrohydrocarbon complexes ; and, as some discrepancies were observed on comparison of the ultra-violet absorption spectra and X-ray photographs of the two hydrocarbons, the identity was not convincingly established.

By subjecting conessine to successive degradations by the Hofmann and Emde processes, Späth and Hromatka ${ }^{3}$ obtained a hydrocarbon, $\mathrm{C}_{21} \mathrm{H}_{30}$, which gave a hexahydroderivative, $\mathrm{C}_{21} \mathrm{H}_{36}$, m.p. 56-58 . In our hands this reduction has yielded approximately equal amounts of two isomeric hydrocarbons, $\mathrm{C}_{21} \mathrm{H}_{36}$, m.p. $56-58^{\circ}$ and $83-84^{\circ}$ respectively. The former is presumably identical with the hydrocarbon ob. tained by Späth and Hromatka, and the isomer, m.p. $83-84^{\circ}$, is identical, chemically and physically, with allopregnane (II), prepared from progesterone by described methods 4 .

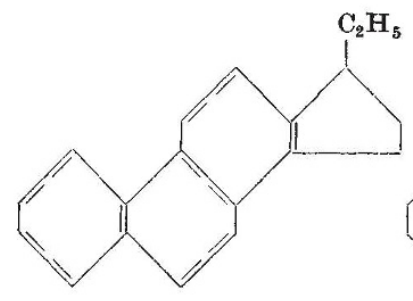

I

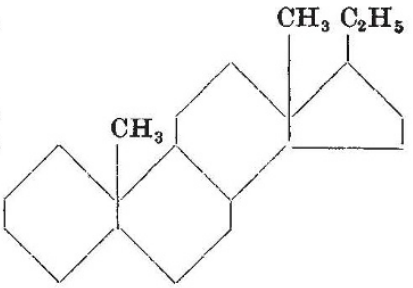

II
The carbon atoms of conessine are therefore accounted for by the allopregnane structure (II) and the three N-methyl groups, but the positions of the ethylenic linkage and the points of attachment of the basic centres are still uncertain. Structural investigations on conessine and its relatives are being developed in several directions, and a full account will be published elsewhere.

\section{R. D. HaWORTH \\ J. McKenNa \\ Nazar Singh}

Department of Chemistry,

University, Sheffield 10. March 22.

${ }^{1}$ Siddiqui and Sharman, Proc. Ind. Acad. Sci., 6 A, 191 (1937).

${ }^{2}$ Riegel, Gold and Kubico, J. Amer. Chem. Soc., 65, 1772 (1943).

Ber., 63, 126 (1930).

- Butenandt and Fleischer, Ber., 68, 2096 (1935). Marker, Kamm Oakwood, Whittle and Lawson, J. Amer. Chem. Soc., 60, 1066 (193\&).

\section{Quantitative Electron-Density Contour Delineation in the Electronic Fourier Synthesizer for Crystal Structure Analysis}

THE electronic Fourier synthesizer for crystal structure analysis reported earlier from this laboratory $^{1}$ has been modified so that contour lines of projected electron densities from one cell on any desired lattice plane can be accurately delineated.

It was originally intended that a pattern corre. sponding to a Bragg photographic synthesis be presented on $a$ cathode ray tube screen; and the remark was made in the early report that such a presentation should not be considered as a replace. ment for accurately calculated contour maps, due to obvious limitations on linearity and range of screen response. This inexactness is removed by triggering coincidence circuits, set at predetermined voltage levels, directly from the voltage signal formerly applied for intensity modulation to the cathode ray tube grid. The circuits produce 1 microsec. pips each time the synthesized signal crosses one of the preset voltage levels, and these are applied to the cathode ray tube grid in place of the entire synthesis signal.

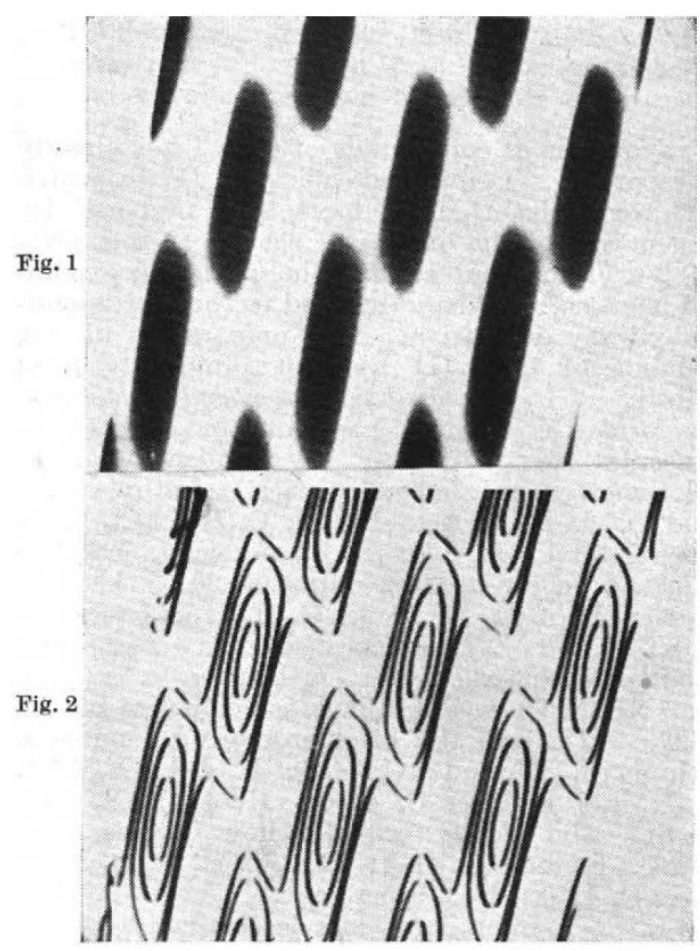

The contouring technique has been applied to the model synthesizer which was demonstrated at the Annapolis meeting of the Crystallographic Society of America on March 20, $1947^{2}$. Fig. 1 shows an intensity-modulated pattern corresponding to the superposition of $(2,0,3)$ and $(1,0, \overline{3})$ fringes, for a $c$-axis projection of a monoclinic cell with $a: c \approx$ $1 \cdot 0: 1 \cdot 1$ and $\beta \approx 81^{\circ}$. Fig. 2 shows a four-line contour map of the same projection. (It may be recalled that the axial ratio and angle of a projection can be adjusted at will on the synthesizer ${ }^{1}$.) These photographs are obtained directly from the presenta. tion tube.

The apparent difference in contours of some peaks in Fig. 2 is due to non-linearity of sweep in the model 